\title{
Using high quality forages to improve in vitro rumen degradability and fermentation of low quality forages
}

M M H Khan, A S Chaudhry

Newcastle University, Newcastle upon Tyne, United Kingdom

Email:m.m.h.khan@ncl.ac.uk

Introduction Low quality forages (LQ), like cereal straws, are the main basal feeds for ruminants in developing countries. However, animal production is low when ruminants are reared on nutrient deficient LQ as the main diet. To get more production from ruminants it is necessary to enhance the utilization of these LQ which could be achieved by using small amounts of high quality forages (HQ) as supplements with LQ. This study compared the effect of incubating in vitro different amounts of various HQ with LQ on the DM degradability (IVD) and fermentation (e.g. ammonia) profiles of rice straw (Straw) and grass hay (Hay) as LQ.

Materials and methods A $3 \times 4 \times 2$ factorial arrangement in duplicate was used to assess the effect of 3 supplements (rye grass $=\mathrm{RG}$, silage $=\mathrm{Si}$ and rape seed plant $=\mathrm{RP}$ ) each at four levels of $0,250,500$ and $750 \mathrm{mg} / \mathrm{g}$ DM on IVD and ammonia (AL) of 2 LQ (Straw and Hay) during 96h of in vitro incubations. Rumen fluid (RF) was obtained from 2 fistulated sheep just before feeding, strained through a cheese cloth, and then it was mixed with a pre-warmed buffer at 1:4 ratio to prepare inoculum. The incubations of forages were conducted in $50-\mathrm{ml}$ polypropylene tubes containing $0.4 \mathrm{~g}$ of ground LQ to which relevant HQ were added according to the experimental design but by enclosing these HQ individually in small polyester bags $(45 \mu)$. These bags permitted the mixing of solubles but not the HQ with LQ and so helped observe the IVD of LQ without their direct mixing with HQ. About $40 \mathrm{ml}$ of the inoculum was added under $\mathrm{CO}_{2}$ to each tube which was sealed with a rubber stopper containing pressure release valve and incubated at $39^{\circ} \mathrm{C}$ for the pre-determined times. After incubation the tubes were submerged in ice to stop fermentation. The liquids and residues were separated by centrifuging the tubes at $3000 \mathrm{rpm}$ for 10 minutes. The residues were washed with distilled water and dried at $60^{\circ} \mathrm{C}$ for $48 \mathrm{~h}$ to determine IVD. The supernatants were acidified with $1 \mathrm{~N} \mathrm{HCl}$ and analysed for AL by using a colorimeter at $660 \mathrm{~nm}$. The IVD and AL data were analyzed by using the General Linear Model of Minitab to study the main effects of LQ, HQ and HQ level=S and their interactions at $\mathrm{P}<0.05$ for the IVD and AL.

Results Main effects of LQ, HQ and $\mathrm{S}$ were significant $(\mathrm{P}<0.003)$ for IVD and AL. However, due to some significant $(\mathrm{P}<0.05)$ interactions between these variables, the mean IVD and AL for each treatment combination are shown in Table 1. IVD was higher for Hay than Straw with all HQ. Mean IVD of LQ was higher with RG than other HQ where IVD increased with RG and Si but reduced with RP for Straw but not for Hay, hence LQ x HQ interaction $(\mathrm{P}<0.002)$. While IVD of both LQ did not increase with the increase in HQ after $250 \mathrm{mg}$, AL continued to increase with increase in HQ from 0 to $500 \mathrm{mg}$. In fact $\mathrm{AL}$ was significantly $(\mathrm{P}<0.001)$ higher for Hay than Straw and it was highest with $500 \mathrm{mg} \mathrm{RP}$ than other HQ.

Table 1 Effects of different level of HQ on the mean IVD and AL for LQ after 96h of incubation

\begin{tabular}{|c|c|c|c|c|c|c|c|c|c|}
\hline \multirow[t]{3}{*}{ LQ } & \multirow[t]{3}{*}{ HQ } & \multicolumn{4}{|c|}{$\operatorname{IVD}(\mathrm{g} / \mathrm{kg})$} & \multicolumn{4}{|c|}{$\mathrm{AL}(\mathrm{mg} / \mathrm{L})$} \\
\hline & & \multicolumn{4}{|c|}{ Amount of HQ (S) (mg/g) LQ } & \multicolumn{4}{|c|}{ Amount of HQ (S) (mg/g) LQ } \\
\hline & & 0 & 250 & 500 & 750 & 0 & 250 & 500 & 750 \\
\hline \multirow[t]{3}{*}{ Straw } & RG & 607 & 666 & 656 & 652 & 92 & 165 & 187 & 188 \\
\hline & $\mathrm{RP}$ & 604 & 583 & 567 & 563 & 96 & 175 & 209 & 205 \\
\hline & $\mathrm{Si}$ & 608 & 633 & 625 & 562 & 93 & 135 & 155 & 168 \\
\hline \multirow[t]{3}{*}{ Hay } & RG & 740 & 765 & 756 & 675 & 124 & 167 & 210 & 212 \\
\hline & $\mathrm{RP}$ & 732 & 738 & 721 & 740 & 120 & 173 & 225 & 220 \\
\hline & $\mathrm{Si}$ & 725 & 808 & 753 & 761 & 128 & 163 & 181 & 182 \\
\hline \multirow{2}{*}{\multicolumn{2}{|c|}{$\begin{array}{l}\text { Pooled SEM } \\
\mathrm{P}<\text { for LQ, HQ, S }\end{array}$}} & \multicolumn{4}{|c|}{11.1} & \multicolumn{4}{|c|}{5.97} \\
\hline & & \multicolumn{4}{|c|}{$\begin{array}{c}\mathrm{LQ}=0.001 ; \mathrm{HQ}=0.002 ; \mathrm{S}=0.003 ; \\
\mathrm{LQxHQ}=0.002 ; \quad \mathrm{LQxS}=0.8 ; \mathrm{HQxS}=0.09 ; \\
\mathrm{LQxHQxS}=0.02\end{array}$} & \multicolumn{4}{|c|}{$\begin{array}{l}\mathrm{LQ}=0.001 ; \mathrm{HQ}=0.001 ; \mathrm{S}=0.001 ; \mathrm{LQxHQ}=0.3 ; \\
\mathrm{LQxS}=0.07 ; \mathrm{HQxS}=0.001 ; \mathrm{LQ} \times \mathrm{HQxS}=0.5\end{array}$} \\
\hline
\end{tabular}

Conclusions The effect of HQ on IVD and AL varied with the type and level of HQ and the LQ during in vitro incubation for 96h. While RP showed negative effect on IVD of LQ, other HQ showed maximum IVD at $250 \mathrm{mg}$. Possible antinutritional factors in RP might have affected the microbial activity which resulted in reduced IVD of LQ. Conversely, more crude protein might have increased AL when HQ were used at higher levels RG and Si can be used as supplements to increase the utilization of LQ but their amounts needs to be optimised in association with the target LQ

Acknowledgements Mr. Khan is grateful to receive UK's ORS award and funding from the Perry Foundation and Sylhet Agriculture University, Bangladesh. 